\title{
An Unusual Case of 32 Weeks Rudimentary Horn Pregnancy with Successful Outcome
}

\author{
Dr. Sonal Bhuyar, Dr Aditi Katkar
}

\section{Introduction}

Mullerian anomalies are often asymptomatic and therefore unrecognized. Unicornuate uterus accounts for $5 \%$ of all mullerian duct anomalies, incidence being approximately 1:4020 women ${ }^{1}$. According to American Fertility Society classification of uterovaginal anomalies Class III B, unicornuate uterus can have presence or absence of rudimentary horn (RH ${ }^{2}$. The presence of $R H$ with cavity leads to well characterized gynecological and obstetrical complications. In 80-90\% of cases, the RH is non-communicating and mostly asymptomatic ${ }^{3}$; however, some contain functional endometrium resulting in chronic pelvic pain, haematometra, endometriosis etc. Pregnancy in a RH is an extremely rare entity, the incidence being 1: 76,000 to 1: 150,000 pregnancies ${ }^{4}$. The natural course of such pregnancy is rupture during first and second trimester, with a potentially life threatening heavy bleeding. Pregnancy in a non-communicating $R H$ with the delivery of a live newborn is even rarer. Ours is a case of RH pregnancy referred to our hospital in the third trimester with successful maternal and fetal survival.

Keywords: Unicornuate uterus, rudimentary horn pregnancy, live birth.

\section{Case Report}

A 25 year old G2A1 was referred to our hospital at 28 weeks in view of severe preeclampsia. She had a spontaneous second trimester abortion in her previous pregnancy. Later she had secondary infertility for which she underwent controlled ovarian hyperstimulation and follicular studies resulting in present pregnancy. Her examination findings showed pedal edema and BP 160/120; however, she had no premonitory symptoms. Obstetrical examination revealed a 26 weeks uterus deviated towards right side with breech presentation and normal fetal heart sounds. She had two conflicting reports of ultrasound with her. One of them was a follicular study report suggesting normal sized uterus with right sided RH with an endometrial thickness of $2 \mathrm{~mm}$. Second was a transvaginal scan done in index pregnancy showing a bicornuate unicollis uterus with right sided horn carrying a 5.4 weeks gestation and attached to left non- gravid horn by a thin band.

Considering no prior history of cryptomenorrhoea or endometriosis and her successful continuation of pregnancy up to 28 weeks, our most probable diagnosis was bicornuate uterus with right horn gravid with preeclampsia and IUGR. Her investigations were within normal limits except 1+ proteinuria and ultrasound confirming IUGR. She was started on treatment for preeclampsia and IUGR with strict maternal and fetal surveillance. But the doses of anti- hypertensive drugs needed frequent escalation due to rising BP. After continuation of pregnancy up to 32 weeks, decision for termination was taken in view of uncontrolled hypertension and Doppler velocimetry showing absent diastolic flow. Patient received two doses of betamethasone for fetal lung maturity. Elective cesarean section was done delivering a male baby by breech weighing 950 grams. Apgar score of $7 / 10$ and $8 / 10$ was noted at $1 \mathrm{~min}$ and $5 \mathrm{~min}$ and baby was shifted to NICU. After delivery of the baby, it was found that there was no communication of right sided gravid horn either with the cervix or the left horn. Left horn was communicating with the cervix which was confirmed by uterine sounding. Bilateral adnexa were normal and attached to their respective horns. Hence the diagnosis of noncommunicating RH was confirmed. After obtaining an informed consent, the RH was excised preserving right ovary and left horn with its adnexa. Postoperative period was uneventful Patient was discharged on $8^{\text {th }}$ day on anti- hypertensive drugs. Baby was discharged at $1.5 \mathrm{~kg}$ weight after 45 days stay in NICU.

\section{Discussion}

Unicornuate uterus with $\mathrm{RH}$ is an uncommon type of mullerian duct malformation. It results from failure of complete development of mullerian duct and incomplete fusion with the contralateral side. Pregnancy in a RH was first described in 1669 by Mauriceau ${ }^{5}$. It is postulated that pregnancy in a non-communicating RH could occur due to transperitoneal migration of the spermatozoon or the fertilized ovum through contralateral side. In the majority of cases, RH ruptures in second trimester; gestation of rupture varies depending on horn musculature, thickness and distensibility of myometrium. IUGR, oligohydramnios, fetal malpresentation, placenta accreta and failed second trimester termination or uterine evacuation represent other forms of presentation of this condition. 
Early diagnosis of the RH pregnancy is essential, but can be challenging. An early bimanual palpation showing a deviated uterus with a palpable adnexal mass extending outside the uterine angle (Baart de la faille's sign) should lead to a suspicion of this anomaly ${ }^{6}$. The availability of advanced ultrasound and magnetic resonance imaging makes the diagnosis of this condition possible at an early gestational age. Tsafrir et al suggested ultrasound criteria of early diagnosis of this condition that include (1) a pseudopattern of asymmetric bicornuate uterus, (2) absent visual continuity between the cervical canal and the lumen of the pregnant horn and (3) presence of myometrial tissue surrounding the gestational $\mathrm{sac}^{7}$.

Pregnancy in a non-communicating RH resulting in a viable fetus is very rare. It is recommended by most that immediate surgery be performed whenever a diagnosis of pregnancy in a $\mathrm{RH}$ is made even if unruptured to avert the catastrophe. In our case, the history of spontaneous abortion, secondary infertility, IUGR, malpresentation and sonography findings favoured the diagnosis of pregnancy in a RH. However, as patient presented to our hospital in third trimester, efforts for continuation of pregnancy to viability were taken.

\section{Conclusion}

Pregnancy in a RH carries a grave risk to the mother. There is a need for increased awareness of this rare condition and to have a high index of suspicion, especially in high risk groups, such as previous history of pelvic pain or infertility, recurrent miscarriages, preterm labour, malpresentation or failure of termination of pregnancy.

\section{Abreviations RH- Rudimentary Horn}

\section{IUGR- Intra Uterine Growth Restriction}

NICU- Neonatal Intensive Care Unit

\section{References}

[1]. Reichman D, Laufer MR, Robinson BK, "Pregnancy outcomes in unicornuate uteri: a review," Fertil Steril 2009 May; $91(5)$ : 188694.

[2]. American Fertility Society classification of mullerian anomalies. Fertil Steril 1988; 49:952.

[3]. Liu MM. Unicornuate uterus with rudimentary horn. Int J Gynecol Obstet. 1994; 44:149-53 (PubMed).

[4]. Nahum GG. Rudimentary uterine horn pregnancy: a case report on surviving twins delivered eight days apart. J Reprod Med. 1997; 42:525-53 (PubMed).

[5]. Jayasinghe $\mathrm{Y}$ et al. The presentation and early diagnosis of the rudimentary uterine horn. Obstet Gynecol. 2005; 105:1456-67 (PubMed).

[6]. Chowdhary S. et al. Pregnancy in a non-communicating rudimentary horn: a clinical case report. Bangladesh Medical Journal. 2010; 39(1):47-48.

[7]. Tsafrir A et al. Rudimentary horn pregnancy: first trimester prerupture sonographic diagnosis and confirmation by MRI. J Ultrasound Med. 2005; 24(2):219-23.

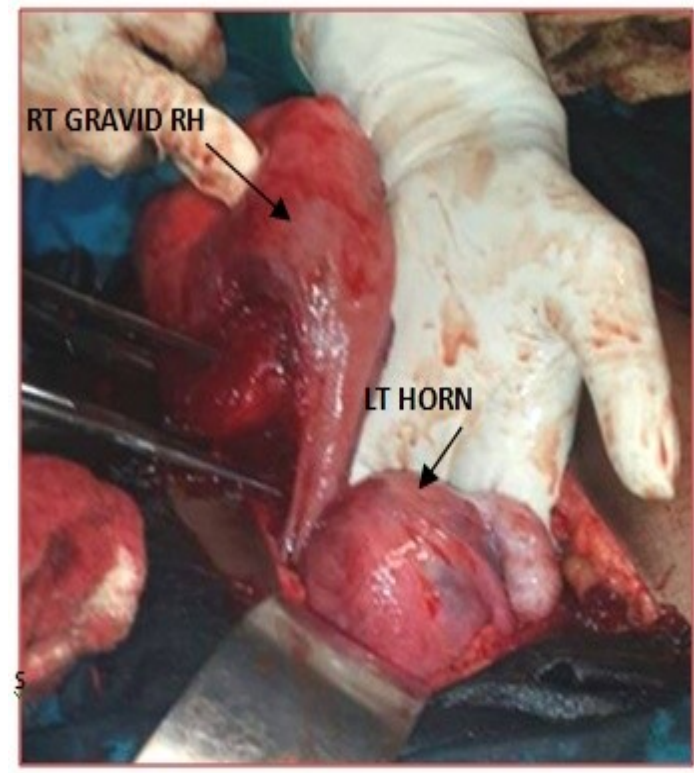

Figure 1 Right gravid rudimentary horn and left nongravid horn with adnexa

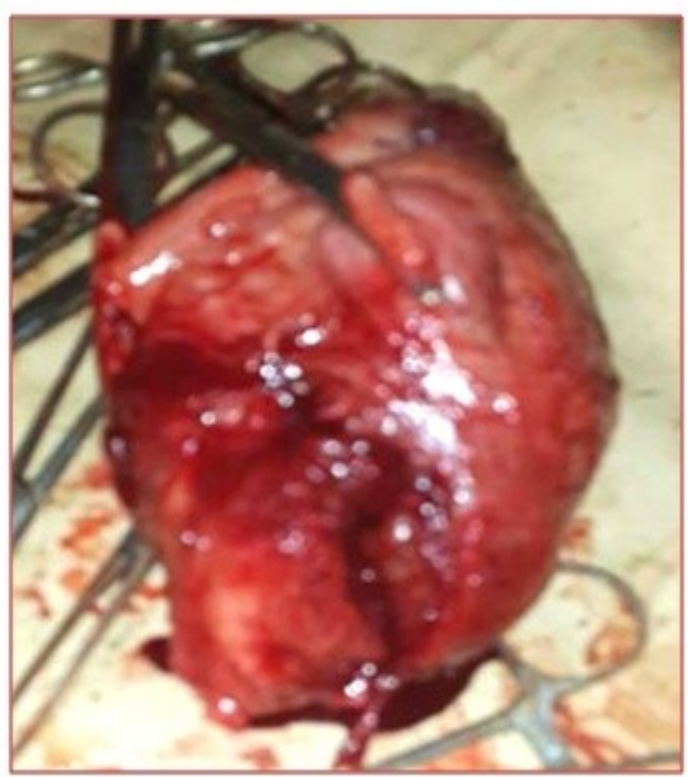

Figure 2 Excised rudimentary horn 


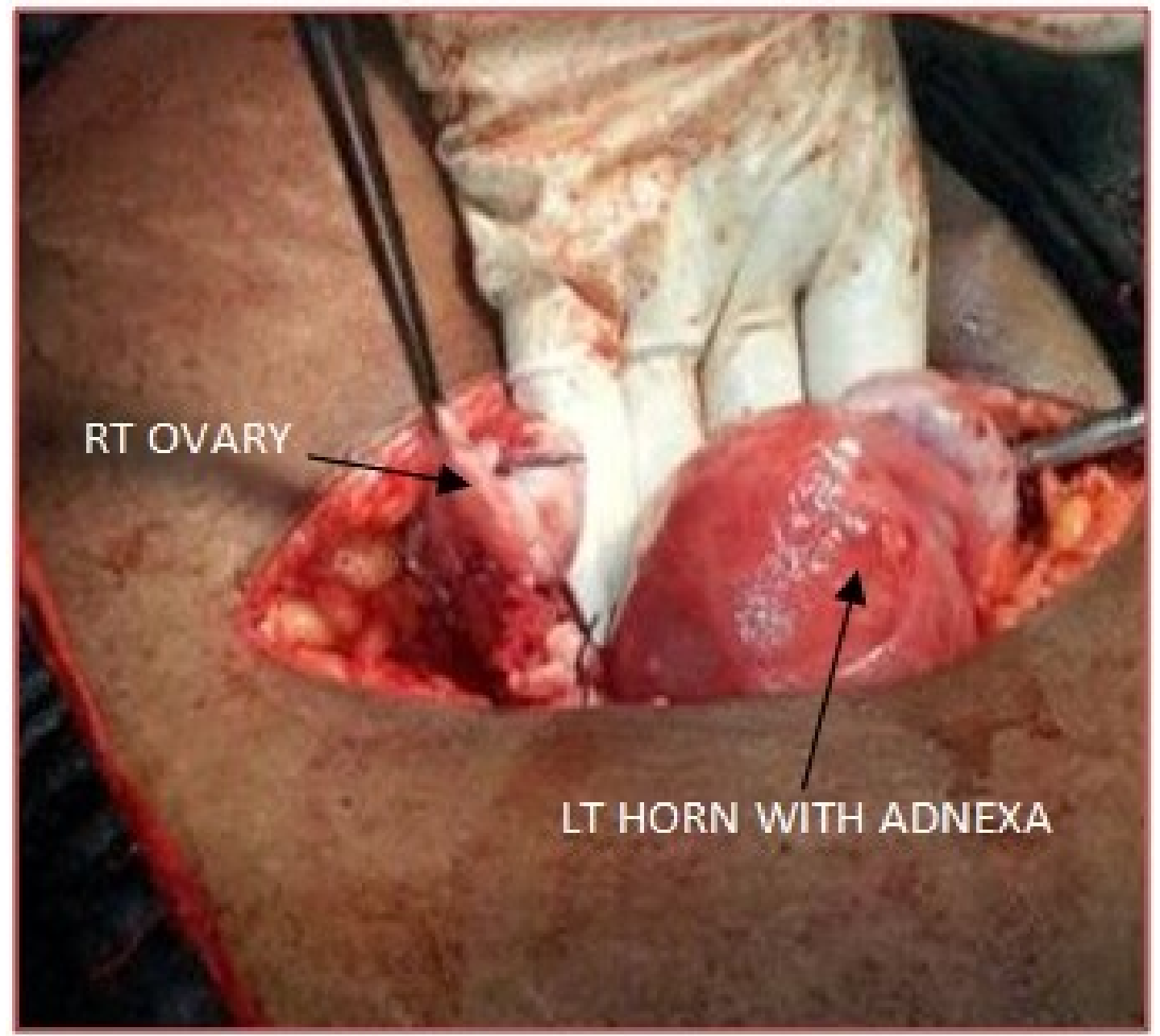

Figure 3 Preserved right ovary and left horn with its adnexa 